\title{
Planning and Implementing Telepsychiatry in a Community Mental Health Setting: A Case Study Report
}

\author{
Hossam Mahmoud $^{1,2} \cdot$ Hady Naal $^{3}(1) \cdot$ Snezana Cerda ${ }^{4}$ \\ Received: 6 June 2020 / Accepted: 2 September 2020 / Published online: 8 September 2020 \\ (c) Springer Science+Business Media, LLC, part of Springer Nature 2020
}

\begin{abstract}
Healthcare institutions in the United States are increasingly adopting telehealth services given their numerous benefits in enhancing access to care. Despite that, few accounts of such organizational experiences in the literature exist, especially those pertaining to telepsychiatry. In this case study, we report the planning and implementation of a telepsychiatry program adopted by a community mental health organization in suburban Chicago, Illinois from 2017 until 2019. We analyze findings gathered from the organization's secondary archival data, highlighting process and outcome evaluations of the program. Results show high levels of patient engagement compared to in-person service modality. Also, our results show an increase in the number of patients served, efficiency in service delivery, decreases in patient wait time to accessing services, and overall positive feedback from patients, families, and staff members. We discuss the successes and challenges encountered by the organization and synthesize them into practical applications recommended for similar initiatives.
\end{abstract}

Keywords Telepsychiatry $\cdot$ Program implementation $\cdot$ Community mental health $\cdot$ Access to care

\section{Introduction}

\section{Background and Problem Statement}

Many healthcare facilities have been turning to telepsychiatry, a web-based videoconferencing modality for the delivery of mental health services, as a means to enhance access to mental health care (Spivak et al. 2019). Seeing the increased demand for mental health services, along with the multiple access barriers, telepsychiatry has become a more attractive

Hady Naal

hady.naal@gmail.com

Hossam Mahmoud

hossam@regrouptelehealth.com

Snezana Cerda

ncerda@josselyn.org

1 Insight+Regroup Telehealth, 4525 Ravenswood Ave \#201, Chicago, IL 60640, USA

2 Tufts University School of Medicine, Boston,, USA

3 Global Health Institute at the American University of Beirut, Beirut, Lebanon

4 The Josselyn Center, 405 Central Ave, Northfield, Chicago, IL 60093, USA option, due to a combination of increased acceptability, feasibility and cost-effectiveness (Chan et al. 2015; Hossam Mahmoud and Vogt 2019; Hossam Mahmoud, Vogt, Dahdouh, and Raymond, 2020; Naal et al. 2020; Spivak et al. 2019). This has been further highlighted during the outbreak of the COVID-19 pandemic, in which the importance of telepsychiatry became even more pronounced (Whaibeh et al. 2020; Peter Yellowlees et al. 2020). In fact, according to recent data, a staggering $4347 \%$ increase in telehealth videoconference use was noted as a response to the limitations imposed by the COVID-19 (Gelburd 2020). Despite that, significant challenges associated with its implementation continue to be reported (Hossam Mahmoud et al. 2019), and more reports are needed to document the planning and implementation experiences of successful telepsychiatry programs, in order to examine associated processes and factors and to facilitate adoption.

The Josselyn Center is a community mental health center located in a suburb of Chicago, Illinois, that provides comprehensive outpatient mental health services, regardless of ability to pay, or insurance coverage, and serves a diverse patient population, many of which are diagnosed with severe and persistent mental illness. Like many healthcare facilities, the Center was facing significant challenges meeting the mental health needs of their patient population, due to the 
shortage of psychiatrists and other providers. In addition, the fact that many patients were uninsured, underinsured, or had public insurance, limited funding to hire a full-time psychiatrist. Furthermore, the shortage of psychiatrists and other providers, particularly within this region, made it difficult to recruit new psychiatric prescribers. In addition, there was a clinician-demographic mismatch, as it was a challenge finding clinicians interested in community mental health work, particularly in that suburban setting, where clinicians may prefer private practice instead. Given these challenges, the center decided to implement a telepsychiatry program.

The aim of this report is to examine the implementation of the telepsychiatry program at this center, and discuss the associated challenges, approaches to mitigating them, outcomes, and lessons learned. This report also recommends approaches for the successful adoption of telepsychiatry in community mental health settings.

\section{Exploring Innovative Solutions}

The Center's available data showed that the wait time to see a psychiatrist had reached four months due to the departure of two out of three part-time psychiatrists, and so the priority was to replace their hours. The Josselyn Center formed a working group which included the President, the Director of Clinical Services, and a few board members, to explore telepsychiatry as an approach to enhancing access to care. Telepsychiatry seemed like an opportunity to access a larger pool of clinicians, leading to increased treatment capacity, while maintaining high quality of care. The center could not recruit an in-person psychiatrist due to a combination of (1) an overall shortage of psychiatric prescribers, (2) the fact that many psychiatric prescribers are not enrolled with insurance networks given low reimbursement rates, and (3) the fact that the center is located in a relatively affluent suburb of Chicago, which meant that many psychiatrists in the area were in private practice and taking cash only. These reasons combined made it a difficult process for the Josselyn center to recruit psychiatrists willing to work in community mental health settings. In addition, videoconferencing aligned with the Center's strategy for improving overall efficiency and quality in healthcare services through expanding the use of healthcare technologies, including electronic health record (EHR) and e-prescribing. This was reinforced by the extensive literature documenting the acceptability and effectiveness of telepsychiatry as a method of mental healthcare delivery, with outcomes similar to in-person care (Chan et al. 2015; Hossam Mahmoud and Vogt 2019; Hossam Mahmoud et al. 2019; Salmoiraghi and Hussain, 2015). Telepsychiatry had become more feasible due to improved connectivity, availability, and affordability of technology, both hardware and software (Ray Dorsey and Topol 2016; Spivak et al. 2019). The Center's information technology
(IT) Department was consulted and confirmed that videoconferencing would be feasible, given the infrastructure at the Center, but that further connectivity safeguards would have to be set up.

Despite the limited cost-effectiveness data available, the group anticipated funding the program with a combination of fee-for-service billing, grants, donations, and fundraising events. The leadership team decided to proceed with implementation, with the understanding that the program itself would be further developed and refined.

The Center decided to implement the program in partnership with a telehealth organization experienced in telepsychiatry program implementation and able to manage the technological and clinical components. The Center sought the help of Regroup Telehealth, which provides integrated telemental health services that would ensure collaboration among different healthcare providers while minimizing disruptions to the center's new workflow. This was driven by the evidence in the literature and recommendations supporting telemental health integration (ATA 2013; Fortney et al. 2015; Mahmoud et al. 2020a; Hossam Mahmoud et al. 2019).

\section{Methods}

Several challenges to implementing telepsychiatry programs have been reported by healthcare facilities across the country, including limitations to institutional support, clinician engagement, clinician licensure requirements, reimbursement restrictions, funding, infrastructure, technological challenges, misinformation and misconceptions about telepsychiatry, concerns about cyber security, and patient privacy (Adaji and Fortney 2017; Lokken et al. 2019; Hossam Mahmoud et al. 2019; Statista 2019; Wang and Alexander 2014). The Center encountered similar challenges and developed approaches to mitigate them by program components, including clinician, on-site staff, patient population, and technology.

\section{Program Challenges}

\section{Clinician}

Due to cost considerations, the Center decided to proceed with two Regroup clinicians, an adult psychiatrist and an advanced practice nurse (APN), who would cover the same number of hours that the parting in-person psychiatrists were offering. While it is generally possible to have a hybrid model that includes a combination of in-person psychiatry and telepsychiatry (P. Yellowlees and Shore 2018), the Josselyn center opted specifically for a telepsychiatry program because the recruited teleclinician were not in a position to 
conduct in-person visits in addition given geographical and transportation challenges. The two teleclinicians underwent training on the software and hardware, project plan, clinical emergency management and technology troubleshooting, and mock sessions. The Josselyn Center and Regroup Telehealth developed a clear workflow that ensured full integration of the teleclinicians and clear and regular communication among team members. An emergency protocol was developed for both during and outside sessions, with identified interventions and clear responsibilities. The workflow was documented in a regularly updated project plan.

\section{On-site Staff}

There was some resistance among different staff members due to varying degrees of comfort with technology. In addition, there were significant misconceptions about telepsychiatry, such as concerns about quality care, privacy, effectiveness, and acceptability for patients. Interestingly, the aforementioned misconceptions and hesitations regarding telepsychiatry are similar to those well-documented in the literature (Hossam Mahmoud et al. 2019). The Center conducted training for on-site staff on the software and hardware using mock sessions, to increase their perceived utility and perceived ease of use of the technology, as these are key in increasing satisfaction and use of technology (Hossam Mahmoud et al. 2019). The training followed the American Telemedicine Association (ATA) guidelines (ATA.2013) and included ways to troubleshoot technology issues, such as speed testing, connectivity issues, camera malfunction, microphone issues, and the protocol of allocating them to the responsible staff for assistance, if simple troubleshooting was not appropriate. Key elements to training telehealth navigators also included ways to provide support to the teleclinicians and emergency protocols, in addition to a focus on assessing patients for alcohol or drug use at time of session, whether by behavior or smell, and communicating this with the teleclinician (Hossam Mahmoud et al. 2019).

\section{Patients}

Some patients were reluctant about telepsychiatry, expressing privacy concerns and worries regarding rapport development with teleclinicians, or that the teleclinicians would not be attentive to their needs, despite documented evidence to the contrary (Germain et al. 2010; Hossam Mahmoud et al. 2019). To increase patient acceptability, staff described the telepsychiatry program, the type of services and clinicians, and the workflow. They also addressed questions and concerns and offered office tours along with technology demonstrations. Ultimately, the decision to refer patients to in-person services or telepsychiatry was based on patient preference, as well as recommendations from their therapists or case managers.

\section{Technology}

Regroup Telehealth provided the HIPAA-compliant videoconferencing platform, electronic fax, and encrypted HIPAA-secure email and assisted with technology preparedness and training. Technology preparedness was enhanced by adding a hotspot in case of internet failure. In addition, laptops that were fully charged and updated were used as backup for technology failure or electric power loss. Also, a second office was equipped with telepsychiatry capability as a backup.

\section{Program Implementation}

Initially, all patients that signed up to use telepsychiatry met with the center's medical coordinator in order to allow them to ask questions on telepsychiatry including the flow of the sessions and the check-in processes among others. The office used for telepsychiatry was purposefully located close to the navigator staff to allow for quick responses if needed. In addition, all staff and medical personnel in the center were updated and informed on telepsychiatry to be able to inform patients who expressed any concerns or who needed additional information on it. The day-to-day implementation of telepsychiatry largely mirrorred in-person psychiatry sessions, with the exception of the clinician being located remotely and conducting the sessions online. Finally, the Center expanded their standard informed consent for services in line with the ATA (ATA.2013) and American Psychiatric Association guidelines, to include information on the use of technology in healthcare including videoconferencing. Patients were also informed of the framework of communication outside of sessions, staff response time, and emergency management.

The telepsychiatry program started delivering fully synchronous outpatient services to patients on May 3, 2017. The psychiatrist and APN had a collaborative agreement, as per the State of Illinois regulations. Both teleclinicians used the same EHR and e-prescribing as other on-site psychiatrist, met regularly with on-site staff, and joined clinical team meetings. A telehealth navigator was responsible for managing the videoconferencing component for sessions, vital signs, scheduling appointments, and helping patients exit the session. The telehealth navigator would help patients operate the laptops provided by the Josselyn center, then once the session started, they would walk out of the room. The workflow was maintained as similar to in-person care as possible, including the process of patient check-in, laboratory test ordering and reporting, referral process, ordering medications, refill policies, no-show policy, scheduling 
system, medication, consent forms, and clinical documentation. In the case of an emergency, patients accessed the Josselyn center hotline, and the Josselyn center staff use the same screening protocols to respond to urgent demands for patients receiving telepsychiatry, as for patients receiving in-person care. That said, patients had the option of choosing the in-person or telepsychiatry services, depending on several factors such as clinician availability, wait times, and personal preference.

\section{Results}

Data for the telepsychiatry program were obtained from reviewing human resources billing records of the Center, between May 3, 2017 and August 28, 2019 (Table 1). During this period the program delivered over $2304 \mathrm{~h}$ of direct patient care, averaging about $19 \mathrm{~h}$ per week, and treating 452 patients on an ongoing basis, over 2931 sessions. Importantly, the waitlist was reduced from 4 months in May 2017, to an average of 1 month in August 2019. Patient engagement rates appeared to be higher for the telepsychiatrist (75\%) compared to the in-person psychiatrist (69\%). Also, telepsychiatry services delivered by the psychiatrist (1.68) showed higher efficiency rates compared to in-person psychiatry (1.17). The aggregate outcomes reflecting the implementation of telepsychiatry services showed them to be more efficient (efficiency rates 1.27 compared to 1.17) than in-person therapy, with comparable patient engagement (70\% show rates compared to $69 \%$ respectively).

Post-session, patients were provided the option of completing a self-reported survey on the computer used to conduct the videoconferencing session, rated on a 5-point Likert scale. Since we reviewed the Center's archival data to develop this case study report, no ethics clearance was required to analyze and report the data. Eighty-nine postsession satisfaction surveys were completed between May 3, 2017 and August 28, 2019 (Table 2). The low response rates of the above surveys and other potential biases, such as comfort with technology, the use of a very brief self-report survey, and possible response bias, make it difficult to fully assess the degree of patient satisfaction. However, anecdotal feedback reported by the Josselyn Center staff involved in the program, such as telehealth navigator, psychotherapists, case managers, and the Clinical Operations Manager, indicated that patients and their families reported overwhelmingly positive feedback, leading to further expansion of the telepsychiatry services. Also, because the same individuals may have responded multiple times, this may influence potential misinterpretations of the data, as they seem to be positively skewed.

While the authors are unable to disclose detailed data on cost-effectiveness, the program has been financially sustainable due to a combination of fee-for-service billing and other
Table 1 Outcome data from May 3rd, 2017 until August 28th, 2019

Table 2 Self-reported patient survey

\begin{tabular}{llll}
\hline Outcome data & Telepsychiatrist & Tele-APN & $\begin{array}{l}\text { In-person } \\
\text { psychia- } \\
\text { trist }\end{array}$ \\
\hline Number of sessions completed & 902 & 2029 & 3915 \\
Total number of patients served & 112 & 340 & 527 \\
Total number of patient-care hours & 537 & 1767.5 & 3349.5 \\
Efficiency of services $^{\mathrm{a}}$ & 1.68 & 1.15 & 1.17 \\
Show rate $^{\mathrm{b}}$ & $75 \%$ & $68 \%$ & $69 \%$ \\
Waitlist to see prescriber (prior to May 3rd, 2017) $^{\text {Waitlist to see prescriber (as of August 28th, 2019) }}$ & 4 months & & \\
\hline
\end{tabular}

${ }^{a}$ Efficiency of services $=$ Number of sessions billed $/$ number of hours staffed

${ }^{\mathrm{b}}$ Show rates $=$ Number of completed sessions $/$ number of sessions completed + number of failed sessions ${ }^{\mathrm{c}}$

${ }^{c}$ Failed sessions $=$ no shows, late cancelations, and cancelations that could not be swapped with an appointment for another patient

\begin{tabular}{lc}
\hline Survey question & $\begin{array}{l}\text { Strongly } \\
\text { agree or } \\
\text { agree }\end{array}$ \\
\hline I feel satisfied with how my telepsychiatry session went today & $98 \%$ \\
The provider answered my questions and addressed my concerns & $99 \%$ \\
Before seeing this provider, I always took my medications the way they were prescribed to me & $86 \%$ \\
After seeing this provider, I am more likely to take my medications as prescribed & $86 \%$ \\
\hline
\end{tabular}


sources of funding, leading the Center to plan an expansion of the telepsychiatry program. The telepsychiatry services have been reimbursed by Medicare, Medicaid, managed care organizations, and increasingly by private insurance. In Illinois, a technology "facility fee" for telepsychiatry services has helped supplement the relatively low Medicaid reimbursement rates for psychiatric services (HFS 2010). In addition, the program has been supported through grants specific for telepsychiatry, donations from the community and board members, and fundraising activities for telepsychiatry funding.

\section{Discussion}

This report describes the planning, implementation, and evaluation of a telepsychiatry program applied within a community mental health center in suburban Illinois. It also discusses different approaches to mitigating challenges to implementations related to technology, patient and staff resistance, and clinician shortage. To our knowledge, this is one of few case studies reporting such an initiative and thus presents a timely contribution to the literature (Hossam Mahmoud et al. 2020a, b; Peter Yellowlees et al. 2020). The value of telepsychiatry became exponentially more evident following the COVID-19 pandemic, and it is expected that more healthcare institutions will start adopting this modality to deliver mental health care moving forward (Naal et al. 2020; Whaibeh et al. 2020; Peter Yellowlees et al. 2020). Our process and outcome evaluations reported in this study may, therefore, serve as a guide for clinicians and program managers looking to integrate telepsychiatry into their clinical programs. The Josselyn Center considers this telepsychiatry program a success, based on the number of patients served, the efficiency of services, the decrease in wait times, the high engagement rates, reported patient satisfaction, and the anecdotal positive feedback received from patients, families and staff (see case vignette for example in Table 3), most of which align with the literature documenting the effectiveness of telepsychiatry (Salmoiraghi and Hussain 2015). Although there does not yet exist a common standardized framework for evaluation of telepsychiatry programs to establish effectiveness, common clinical and non-clinical outcomes have been used for this purpose. For example, it has been consistently demonstrated using standardized clinical measures that telepsychiatry is associated with a plethora of treatment outcomes that are comparable to in-person treatment, with the added benefit of increased access to mental health care (Hilty et al. 2014; Wang and Alexander, 2014).

The planning and implementation processes of this program have utilized many of the "core competencies" described in the literature as critical for telehealth implementation, including the utilization of implementation services that help in planning, training, education and implementation, as well as operations support, regulatory compliance, and teleclinician management (Adaji and Fortney 2017; Lokken et al. 2019; H Mahmoud et al. 2020a, b; Naal et al. 2020; Wang and Alexander 2014). While some of these services could have been implemented in-house, such as ensuring adequate support through a telehealth navigator and other clinical services, there was a significant value to partnering with a telepsychiatry organization that has experience with telepsychiatry implementation, for ongoing issue tracking, teleclinician training, teleclinician management, compliance, and legal support. Several other factors have contributed to the success of the program, and

Table 3 Case vignette

\section{Case vignette of an older patient with depression and anxiety}

The Josselyn Center introduced the option of telepsychiatry to its patient population as a solution to the different access challenges to receiving care. While some patients readily embraced this transition, others expressed initial skepticism. For example, one patient aged over 70 , with chronic and severe depression and anxiety, and who was on a fixed income had been seeing the same psychiatrist at the center in person for over 10 years. That psychiatrist was retiring, and the patient did not warm up to the idea of changing his psychiatrist, and not to the notion of conducting his sessions via telepsychiatry

However when reviewing his options, the patient realized that he would have to wait over 3 months to be able to see another in-person psychiatrist at the Center or possibly wait several months to be able to see a new psychiatrist outside the Center, the latter not being feasible due to the patient's transportation limitations. In fact, the uncertainty about being able to continue treatment without interruption worsened his anxiety and depressive symptoms. Telepsychiatry seemed like an increasingly more appealing option for him, that would allow him to avoid care interruption and maintain his connection with the Center and its staff, which he was familiar and comfortable with

After his first telepsychiatry session, this patient was pleasantly surprised with how smoothly it went, and reported excitement with having a "techy session" which he went on to share with his children. He also described forgetting that he was communicating through a screen, since the provider was warm and engaging. In addition, the patient was pleasantly surprised that the telepsychiatrist was coordinating his treatment with the other members of his treatment team, despite the remote location of the psychiatrist. The patient engaged in treatment, developed a strong therapeutic alliance with the psychiatrist, and experienced a significant and sustained improvement in his symptoms

The patient continues his telepsychiatry sessions on a regular basis, and he engages in other therapeutic services regularly. He was initially being seen on a monthly basis, but as his symptoms improved, his telepsychiatry sessions for routine medication monitoring were spaced out to every 3 months 
we recommend that other healthcare organizations looking to implement telepsychiatry programs take them into consideration:

- Integrating the services and utilizing a collaborative approach to care.

- Ensuring technology preparedness, with a contingency plan for technology breakdown.

- Training on-site staff on the workflow, technology, teleclinician support, and emergency protocols.

- Having a clear project plan, that describes the workflow and responsibilities of every member involved in the project, including lead role allocation for every aspect of the program.

- Establishing clear communication standards between the Center, telehealth organization, and the teleclinician to ensure that any issues are addressed in a timely manner.

The next phase of this program has included expansion of telepsychiatry services as of September 2019 with an emphasis on yet another core competency, data analytics, which has not been at the center of the first phase of the program. Data analytics will be an integral part of the program, with monitoring of such metrics as degree of utilization, clinician satisfaction and patient satisfaction, and return on investment in order to improve the program (Lokken et al. 2019). Patient satisfaction will be examined with a more indepth assessment of elements contributing to degree of satisfaction, including questions on appointment promptness, clinically-appropriate timeframe for follow up appointment, and response to refill requests. Other measures will examine self-reported medication adherence, which would be verified by laboratory testing or pharmacy records.

Also, the next phase will include the introduction of standardized assessment tools such as the Patient Health Questionnaire-9 (PHQ-9) and Generalized Anxiety Disorder-7 (GAD-7). Additionally, regularly scheduled peer reviews that incorporate 2019 Merit-based Incentive Payment System (MIPS) quality measures (Healthmonix 2019) will be added. The ongoing data monitoring will serve as a quality assurance measure, in order to continuously fine tune the program. Finally, tracking of costs including general overhead, staffing costs and technology costs of the telepsychiatry program will need to be incorporated, and factoring in patient retention and payor reimbursement, especially as the program is expanded.

\section{Conclusion and Future Recommendation}

As telepsychiatry programs continue to expand across the country, yet continue to face significant implementation challenges, implementation reports such as this one aim to offer guidance on developing and sustaining a successful telepsychiatry program, by detailing considerations, challenges, and impact of the program. Future research is advised to examine the long-term community-level impact of such programs, with regards to their potential to enhance access to mental health services and to reduce the burden of mental disorders in given settings.

Author Contributions All authors contributed to the study conception and design. Material preparation and data analysis were performed by HM and HN. The first draft of the manuscript was written by HM and $\mathrm{HN}$ and all authors commented on previous versions of the manuscript. All authors read and approved the final manuscript.

Funding This research received no specific grant from any funding agency in the public, commercial, or not-for-profit sectors.

\section{Compliance with Ethical Standards}

Conflict of interest H.M is the Medical Director of Regroup Telehealth, and S.C is the Director of the Josselyn Center. The publication or lack thereof, of this manuscript will neither positively nor negatively influence their positions, salaries, promotion, or other aspects related to their work at Regroup or the Josselyn Center.

Ethical Approval This study used secondary archival data of the Josselyn center, therefore no ethics approval was required.

\section{References}

Adaji, A., \& Fortney, J. (2017). Telepsychiatry in integrated care settings. Focus, 15(3), 257-263. https://doi.org/10.1176/appi.focus .20170007 .

ATA. (2013). Practice guidelines for video-based online mental health services. Telemedicine and e-Health, 19(9), 722-730.

Chan, S., Parish, M., \& Yellowlees, P. (2015). Telepsychiatry today. Current Psychiatry Reports, 17(11), 1-9. https://doi.org/10.1007/ s11920-015-0630-9.

Fortney, J. C., Pyne, J. M., Turner, E. E., Farris, K. M., Normoyle, T. M., Avery, M. D., et al. (2015). Telepsychiatry integration of mental health services into rural primary care settings. International Review of Psychiatry, 27(6), 525-539. https://doi. org/10.3109/09540261.2015.1085838.

Gelburd, R. (2020). FAIR Health's monthly telehealth regional tracker suggests impact of COVID-19: Telehealth claim lines increase 4347\% Nationwide in a year. Retrieved August 1, from https:// www.ajmc.com/view/fair-healths-monthly-telehealth-regionaltracker-suggests-impact-of-covid19-telehealth-claim-lines-incre ase-4347-nationwide-in-a-year.

Germain, V., Marchand, A., Bouchard, S., Guay, S., \& Drouin, M. S. (2010). Assessment of the therapeutic alliance in face-to-face or videoconference treatment for posttraumatic stress disorder. Cyberpsychology, Behavior, and Social Networking, 13(1), 29-35. https://doi.org/10.1089/cyber.2009.0139.

Healthmonix. (2019). Mental/behavioral health MIPS specialty measure set. Retrieved May 30, from https://healthmonix.com/mips_ measure_set/mental-behavioral-health-mips-specialty-measu re-set-2019/. 
HFS. (2010). Expansion of telehealth services. Retrieved May 30, from https://www.illinois.gov/hfs/MedicalProviders/notices/Pages/ prn100112a.aspx.

Hilty, D. M., Yellowlees, P. M., \& Nasatir, S. E. (2014). Program evaluation and practical, step-by-step program modification in telemental health. Behavioral telehealth series volume 1-Clinical video conference: Program development and practice (pp. 105-134). New York: Springer Press.

Lokken, T. G., Blegen, R. N., Hoff, M. D., \& Demaerschalk, B. M. (2019). Overview for implementation of telemedicine services in a large integrated multispecialty health care system. Telemedicine and E-Health, OO(00), 1-6. https://doi.org/10.1089/tmj.2019.0079.

Mahmoud, H., \& Vogt, E. (2019). Telepsychiatry: an innovative approach to addressing the opioid crisis. Journal of Behavioral Health Services and Research, 46(4), 680-685. https://doi. org/10.1007/s11414-018-9611-1.

Mahmoud, H., Vogt, E. L., Dahdouh, R., \& Raymond, M. L. (2020a). Using continuous quality improvement to design and implement a telepsychiatry program in rural Illinois. Psychiatric Services. https://doi.org/10.1176/appi.ps.201900231.

Mahmoud, H., Vogt, E. L., Sers, M., Fattal, O., \& Ballout, S. (2019). Overcoming barriers to larger-scale adoption of telepsychiatry. Psychiatric Annals, 49(2), 82-88. https://doi.org/10.3928/00485 713-20181228-02.

Mahmoud, H., Whaibeh, E., \& Mitchel, B. (2020b). Ensuring successful telepsychiatry program implementation: Critical components and considerations. Current Treatment Options in Psychiatry, 7(2), 186-197.

Naal, H., Whaibeh, E., \& Mahmoud, H. (2020). Guidelines for primary health care-based telemental health in a low-to middle-income country: The case of Lebanon. International Review of Psychiatry. https://doi.org/10.1080/09540261.2020.1766867.

Ray Dorsey, E., \& Topol, E. J. (2016). State of telehealth. New England Journal of Medicine, 375(2), 154-161. https://doi.org/10.1056/ NEJMra1601705.
Salmoiraghi, A., \& Hussain, S. (2015). A systematic review of the use of telepsychiatry in acute settings. Journal of Psychiatric Practice, 21(5), 389-393. https://doi.org/10.1097/PRA.0000000000 000103.

Spivak, S., Spiva, A., Cullen, B., Meuchel, J., Johnston, D., Chernow, R., et al. (2019). Telepsychiatry use in US mental health facilities. Psychiatric Services, 71(2), 121-127. https://doi.org/10.1176/ appi.ps.201900261.

Statista. (2019). Which of the following has been a challenge to implementing telemedicine practices in your organization? Retrieved May 30, from https://www.statista.com/statistics/870073/telem edicine-program-implementation-challenges/.

Wang, L., \& Alexander, C. A. (2014). Telepsychiatry: Technology progress, challenges, and language and transcultural issues. Journal of Translational Medicine and Developmental Disorders, 1(1), 1-11. https://doi.org/10.12691/jtmdd-1-1-1.

Whaibeh, E., Mahmoud, H., \& Naal, H. (2020). Telemental health in the context of a pandemic: The COVID-19 experience. Current Treatment Options in Psychiatry. https://doi.org/10.1007/s4050 1-020-00210-2.

Yellowlees, P., Nakagawa, K., Pakyurek, M., Hanson, A., Elder, J., \& Kales, H. C. (2020). Rapid conversion of an outpatient psychiatric clinic to a $100 \%$ virtual telepsychiatry clinic in response to COVID-19. Psychiatric Services, 71(7), 749-752. https://doi. org/10.1176/appi.ps.202000230.

Yellowlees, P., \& Shore, J. (2018). Telepsychiatry and health technologies: A guide for mental health professionals (1st ed.). Washington: American Psychiatric Publications Incorporated.

Publisher's Note Springer Nature remains neutral with regard to jurisdictional claims in published maps and institutional affiliations. 